\title{
Una aproximación semántica a la filosofía teórica de Kant
}

\section{A semantical approach to Kant's theoretical philosophy}

\author{
Àlex MumBrú MorA \\ Universidad de Barcelona
}

Recibido: 29-09-2011

Aceptado: 29-02-2012

\section{Resumen}

A pesar de las escasas referencias al lenguaje en la obra de Kant, cabe plantear la existencia de una concepción semántica latente en el conjunto de la filosofía trascendental. La cuestión del significado se corresponde en Kant a la pregunta por el fundamento de la relación entre una representación universal (o tipo) y el particular que le corresponde. De acuerdo con lo presentado en el parágrafo 59 de la Crítica de la facultad de juzgar, dos son los modelos que nos permiten pensar toda posible relación particular-tipo (hipotiposis): la exposición esquemática y la exposición simbólica.

El objetivo principal del presente artículo es analizar la naturaleza, función y lugar sistemático de la noción de exposición esquemática en cuanto modelo de significación operante en el dominio teórico, mostrando a su vez la crítica implícita a la comprensión del funcionamiento de nuestros conceptos en autores como Locke, Berkeley y Hume. Se ubicará asimismo el concepto de exposición simbólica en el contexto de la problemática de una exposición de las ideas de razón.

Palabras clave: lenguaje, significado, hipotiposis, exposición esquemática, exposición simbólica.

\begin{abstract}
Despite the limited references to language in Kant's work, there is an implicit conception of meaning in trascendental philosophy as a whole. The question about
\end{abstract}


meaning is equivalent to the question about the basis of the relation between a universal representation (or type) and the token which corresponds to it. In the 59th paragraph of the Critique of Judgement, Kant considers two models which allow us to think all possible type-token relations (hypotyposis): the schematic and the symbolic exposition.

The main aim of our paper is to analyze the nature, function and systematic place of the schematic exposition as the signification model that applies in the theoretical domain. We will also bring about Kant's implicit criticism of the understanding of the functioning of our concepts, as found in Locke, Berkeley and Hume. A brief glimpse into the notion of symbolic exposition will be given as well, by pointing at the problem of an exposition of our ideas of reason.

Keywords: language, meaning, hypotyposis, schematic exposition, symbolic exposition.

\section{Introducción}

Dos son las líneas mayores de interpretación del pensamiento kantiano y, en particular, de la Crítica de la razón pura $(\mathrm{KrV})$ en cuanto obra fundacional del período crítico en Kant y de la filosofía trascendental en cuanto tal ${ }^{1}$. Nos encontramos por un lado con aquellos autores que subrayan el carácter eminentemente epistemológico de la filosofía trascendental, entendiendo la $\mathrm{KrV}$ como un proyecto de fundamentación del conocimiento científico de la naturaleza ${ }^{2}$. Por otro, aquellas aproximaciones que consideran que el objetivo fundamental del pensamiento crítico en su conjunto es un examen de la validez de los principios de la metafísica tradicional (Dios, alma, mundo), encaminado a dar cuenta -y, en la medida de lo posible, a subsanar- del proceso de dialéctica autodestrucción que ha padecido 3 . Ahora

\footnotetext{
${ }^{1}$ Como es habitual, la citación de los textos kantianos sigue la edición de la Academia (Ak), consignando el volumen en números romanos y la página en numeración arábiga, a excepción de la Crítica de la razón pura $(\mathrm{KrV})$ que se cita de acuerdo con el procedimiento usual, según la paginación de las ediciones A o B. Las traducciones de los textos de Kant son del autor de este artículo.

2 Entre los estudios clásicos de esta perspectiva epistemológica deben destacarse las obras de los neokantianos H. Cohen, Kants Theorie der Erfahrung (1871) y E. Cassirer, Das Erkenntnisproblem in der Philosophie und Wissenschaft der neueren Zeit (1906), los volúmenes I y II especialmente, así como su Kants Leben und Lehre (1918). En esta misma línea se sitúa la lectura ofrecida por H. J. Paton en Kant's Metaphysics of Experience (1961), por la que la metafísica pasa a concebirse en términos de aquella disciplina encargada de describir las condiciones que hacen posible todo conocimiento de la naturaleza, de tal modo que puede hablarse del proyecto trascendental como de una «metafísica de la experiencia». Con las matizaciones que señalaremos más adelante, cabe ubicar también la obra de P. Strawson The bounds of sense (1975) en esta línea general de lectura.

3 Desde la publicación de la primera crítica, asistimos a una profunda transformación de la arquitectónica del sistema esbozada por Kant (KrV, B860-880/A832-852). Es en la obra de 1791, Los avances
} 
bien, planteadas en estos términos, ambas líneas interpretativas son del todo complementarias; así, la reflexión sobre la posibilidad de un conocimiento matemático de la naturaleza está esencialmente orientada en Kant a la pregunta por la posibilidad de la metafísica como ciencia, esto es, a la posibilidad de un conocimiento metafísico bajo la restricción crítica de las condiciones a priori de la posibilidad de la experiencia para el ser racional finito 4 .

A partir del giro lingüístico acaecido en la filosofía del siglo XX, a estas dos canónicas estrategias interpretativas del pensamiento de Kant se le ha añadido una tercera; a saber, aquélla que pretende extraer la concepción semántica operante en la filosofía trascendental ${ }^{5}$. Cabe señalar no obstante que la mayor parte de aproximaciones al pensamiento de Kant desde la filosofía analítica del lenguaje se han centrado de manera casi exclusiva en un análisis de la naturaleza semántica de nuestros conceptos empíricos (y, por extensión, del funcionamiento de los juicios de experiencia) en su relación con los esquemas trascendentales de la imaginación6. En

de la metafisica desde los tiempos de Leibniz y Wolff (Fortschritte), donde se produce una primera reformulación de la arquitectónica de la razón sobre la base de la función cuasi-constitutiva de la facultad de juzgar teleológica reconocida por Kant en la Crítica de la facultad de juzgar (KU). Ahora bien, esta reestructuración no supone renuncia alguna a la posibilidad de un cierto tipo de conocimiento de lo suprasensible (libertad, alma, Dios), siendo la metafísica precisamente "aquella ciencia en la que se avanza desde el conocimiento de lo sensible al de lo suprasensible por medio de la razón" [(Fortschritte (Ak, XX, 260)]. Es decir: si bien es cierto que el conocimiento trascendental es una investigación sobre las condiciones a priori de la posibilidad de la experiencia, esta reflexión se encamina hacia una reformulación del discurso metafísico que sea compatible con la posibilidad de un conocimiento del ámbito de lo suprasensible, en un claro avance de las problemáticas de que se ocuparán posteriores desarrollos idealistas; cfr. Turró, S., Tránsito de la naturaleza a la historia en la filosofia de Kant, Barcelona, Anthropos, 1996, §§40-46.

4 En este sentido, es suficientemente revelador el título de la obra de 1783 Prolegómenos a toda metafisica futura que haya de poder presentarse como ciencia (Prolegomena), cuyo principal propósito es exponer de un modo más abarcable y claro tanto los objetivos como los resultados fundamentales de la $\mathrm{KrV}$ en su primera edición.

5 Pueden considerarse los escritos de R. E. Butts, "Kant's Schemata as Semantical Rules" (en L. W. Beck (ed.), Kant's Studies Today, La Salle, Open Court, 1969, pp. 290-300) y de M. Thompson, "Singular Terms and Intuitions in Kant's Philosophy" [Review of Metaphysics (Diciembre de 1972), pp. 314-43], como textos fundacionales de una interpretación de la filosofía de Kant en términos de una teoría del significado. Es en la línea de investigación abierta por estos dos autores, y subrayando la índole eminentemente epistemológica de la filosofía trascendental, donde cabe inscribir la célebre obra de P. Strawson, The bounds of sense (trad. de C. Thiebaut, Madrid, Revista de Occidente, 1975), cuya aportación principal consiste en ofrecer una interpretación semántica global de la filosofía teórica de Kant desde el resultado fundamental de la investigación crítica; a saber, el establecimiento del «Principio de Significatividad» (Principle of Significance) en cuanto conjunto de las condiciones (experimentales) a que deben someterse nuestros conceptos para estar en posesión de un significado cognoscitivamente válido.

6 A parte de los citados en la nota anterior, en esta línea de trabajo cabe destacar: Pippin, R. B., "The Schematism and Empirical Concepts", Kant-Studien, 67 (1976), pp. 156-171; Nolan, J. P., "Kant on Meaning: Two Studies", Kant-Studien, 70 (1979), pp. 113-130; Hanna, R., "Kant's Theory of 
este sentido, en la medida en que se han ocupado fundamentalmente de la filosofía teórica de Kant, cabe afirmar que esta línea de investigación constituye una aproximación en cierto modo subalterna a la comprensión epistemológica de la filosofía trascendental.

Como mostraremos a continuación, los dos modelos de exposición presentados por Kant en el parágrafo 59 de la Crítica de la facultad de juzgar (KU) -la exposición esquemática y la exposición simbólica7- permiten ofrecer una interpretación semántica global de la filosofía kantiana. El objetivo del presente artículo es analizar la naturaleza, función y lugar sistemático del concepto de «exposición esquemática» en cuanto modelo de significación operante en el dominio teórico, si bien apuntaremos algunos de los problemas que derivan de éste en su aplicación a los conceptos de razón.

\section{El papel del lenguaje en la filosofía trascendental kantiana}

La reflexión sobre el significado aparece estrechamente ligada a la cuestión del lenguaje. Ahora bien, Kant se ocupa tangencialmente del elemento lingüístico; además de en algunas de sus Reflexiones, básicamente en la Antropología en perspectiva pragmática (Anthropologie) y en las Lecciones de Metafísica, en el apartado dedicado a la psicología empírica ${ }^{8}$. Es decir: la reflexión sobre la naturaleza y funcionamiento del lenguaje aparece una vez que el trabajo sistemático de fundamentación ha sido ya realizado. Y el hecho de que quede relegado a un lugar extramuros con respecto al complejo de la filosofía trascendental supone no reconocerle valor constitutivo alguno en relación a los tres ámbitos de validez de que se ocupa la filosofía crítica (conocimiento, moral, objeto estético). Es por este motivo que

\footnotetext{
Empirical Judgment and Modern Semantics", History of Philosophy Quarterly, vol. 7, n. 3 (Julio de 1990), pp. 335-351; del mismo autor, "The trouble with Truth in Kant's Theory of Meaning", History of Philosophy Quarterly, vol. 10, n. 1 (Enero de 1993), pp. 1-20; Lohmar, D., „Kants Schemata als Anwendungsbedingungen von Kategorien auf Anschauungen", Zeitschrift für philosophische Forschung, 45 (1991), pp. 77-92; del mismo autor, "Husserl's Type and Kant's Schemata. Systematic Reasons for Their Correlation or Identity", en D. Welton (ed.), The New Husserl, Indiana University Press, 2003, p. 93-124; Düssing, K., „Schema und Einbildungskraft in Kants Kritik der reinen Vernunft", en Günter Gawlick y Lothar Kreimendahl (ed.), Aufklärung und Skepsis. Studien zur Philosophie und Geistesgeschichte des 17. und 18. Jahrhunderts, Stuttgart, Frommann-Holzboog, 1995, pp. 47-71.

7 Cfr. KU, \$59 (Ak, V, 351).

8 Anthropologie, $\$ 38$ (Ak, VII, 191ff) y Vorlesungen über Metaphysik, herausgegeben von K. H. L. Pölitz, Erfurt, 1821, pp. 19 y 158ss. Con respecto al papel que juega el lenguaje en la filosofía de Kant pueden consultarse: Markis, D., „Das Problem der Sprache bei Kant”, en B. Scheer y G. Wohlfart (ed.), Dimensionen der Sprache in der Philosophie des Deutschen Idealimus, Würzburg, Königshausen und Neumann, 1982, pp. 110-154; Villers, J., Kant und das Problem der Sprache, Konstanz, Verlag am Hockgraben, 1997.
} 
Kant no puede conceder al elemento lingüístico más que una función meramente instrumental.

Concebido como uno de los posibles rendimientos de la «facultad de designar» (Bezeichnungsvermögen) en general, el lenguaje constituye un conjunto de signos de carácter artificial por medio de los cuales podemos tanto hacer referencia a los «objetos extralingüísticos» como expresar nuestros propios pensamientos ${ }^{9}$. Ya sea natural o algebraico, el lenguaje es un «signo sensible» (sinnliche Zeichen) de nuestros pensamientos ${ }^{10} \mathrm{y}$, precisamente porque les proporciona visibilidad, es el medio por el que podemos comunicarlos a los demás ${ }^{11}$; así, por lenguaje entiende Kant un sistema de signos que substituyen o están en lugar de los objetos o pensamientos que expresan ${ }^{12}$. No debe identificarse pues el carácter discursivo de nuestro conocimiento -el hecho de estar esencialmente mediado por conceptos- con su carácter lingüistico, precisamente porque nuestros signos lingüísticos y algebraicos constituyen "meras expresiones para conceptos"13.

9 Cfr. Anthropologie, $\$ 38$ (Ak, VII, 191ff). La facultad de designar conforma un ámbito más extenso
que el delimitado por la facultad lingüística, dado que Kant establece tres tipos de «signo»: a) signo
natural; b) signo artificial; c) signos milagrosos (Wunderzeichen). El signo lingüístico constituye un
signo de carácter artificial en la medida en que no encontramos similitud alguna entre el signo y la
imagen del objeto. Precisamente porque el lenguaje no nace de ninguna similitud con el objeto que
designa, es concebible la existencia de una infinidad de lenguas diferentes o, expresado en otros tér-
minos, el carácter convencional del lenguaje; cfr. Markis, D., op.cit., p. 120.
10 “Todo lenguaje es la designación (Bezeichnung) de los pensamientos y, a la inversa, la manera más
excelente de designar pensamientos es a través del lenguaje, el mayor medio para comprenderse a sí
mismo y a los otros"; Anthropologie, §38 (Ak, VII, 192). En este contexto, el término «designar» debe
entenderse en el sentido de que el signo lingüístico constituye una marca sensible por la que es posi-
ble exteriorizar nuestros pensamientos y, por ende, comunicarlos a los demás. 11 "El lenguaje es comunicación de los pensamientos"; Reflexionen (Ak, XVI, Nr. 1620).

12 Una de las tesis de la obra de J. Villers es que Kant no hubiera podido llevar a cabo una reflexión sistemática y coherente sobre la naturaleza del lenguaje sin haber dinamitado uno de los presupuestos fundamentales de su filosofía; a saber, el rígido dualismo establecido entre el entendimiento y la sensibilidad. La facultad de designar (facultas sygnatrix), ubicada en el seno de la imaginación productiva, constituiría la «desconocida raíz común» de los dos troncos del conocimiento: en la medida en que debe conservar la aprehensión unitaria del material dado en la intuición para asociarlo a un signo que ella misma produce, la facultad de designar ocuparía un lugar intermedio entre la receptividad de la sensibilidad y la espontaneidad del entendimiento. En este mismo sentido, la naturaleza del signo lingüístico eliminaría el postulado de la heterogeneidad e irreductibilidad entre la sensibilidad y el entendimiento, en la medida en que todo signo lingüístico es portador sensible de un significado de carácter intelectual.

13 “Ambos [el modo de representación esquemático y el simbólico] son hipotiposis, i.e. exposiciones (exhibitiones), no meros caracterismos; es decir, designaciones de los conceptos por medio de signos sensibles que les acompañan y que no contienen absolutamente nada perteneciente a la intuición del objeto, sino que sirven tan sólo como medio para la reproducción (en intención subjetiva por tanto) conforme a la ley de asociación de la imaginación. Estos signos sensibles son o bien palabras, o signos visibles (algebraicos, incluso mímicos), en tanto que meras expresiones para conceptos"; KU, §59 $(\mathrm{Ak}, \mathrm{V}, 352)$. 
Aun cuando las reflexiones sobre el lenguaje por parte de Kant son prácticamente inexistentes, resulta razonable plantear la existencia de una concepción semántica latente en el conjunto de la filosofía trascendental14. Como consecuencia del carácter meramente instrumental concedido al lenguaje, cabe afirmar que preguntarse por el significado de nuestros términos lingüísticos es tanto como preguntarse por el concepto expresado por tal término, puesto que la suposición de la prioridad del pensamiento sobre el lenguaje implica que el rendimiento cognoscitivo de este último es nulo. La cuestión del significado se corresponde en Kant a la pregunta por el fundamento de la relación entre una representación universal dada y el particular que le corresponde. En este sentido habla Kant de «sensibilización» (Versinnlichung), «exposición» (Darstellung) o «hipotiposis» (Hypotypose) como aquel proceso por el que nos es en general posible proporcionar un contenido intuitivo a una determinada representación conceptual:

toda hipotiposis (exposición, subiectio sub adspectum), en cuanto sensibilización, es doble: o esquemática, cuando a un concepto que es aprehendido por el entendimiento le es dada la correspondiente intuición a priori; o bien simbólica, cuando a un concepto que sólo la razón puede pensar y al que ninguna intuición sensible puede serle adecuada, se le subsume una intuición con respecto a la cual el proceder de la facultad de juzgar coincide de un modo meramente analógico con aquello que observa cuando esquematiza, i.e. en la regla de tal proceder solamente, mas no en la intuición misma; coincide por tanto según la forma de la reflexión meramente, y no en lo que respecta al contenido ${ }^{15}$.

Dos son, pues, los modelos que nos permiten pensar toda posible relación particular-tipo: la exposición esquemática, que articula la relación particular-tipo en el ámbito teórico; y la exposición simbólica, que proporciona cierto correlato sensible a aquellas representaciones conceptuales (las ideas o conceptos de la razón) que escapan por definición a toda sensibilización directa ${ }^{16}$. La cuestión del significado, i.e. la pregunta por el fundamento de la relación entre el concepto en cuanto representación universal y la intuición del objeto en cuanto presentación de un «particular»17, deberá atender pues a la naturaleza de los conceptos que son propios del

14 La expresión «filosofía del lenguaje latente» (latente Sprachphilosophie) es de J. Villers, op.cit., p. 336. Ahora bien, la idea de una concepción del lenguaje implícita en el pensamiento de Kant se encuentra ya en D. Markis, op.cit., p. 111, artículo en que se basa en gran medida la obra de J. Villers. 15 Cfr. KU, §59 (Ak, V, 351).

16 La distinción entre exposición esquemática y exposición simbólica es recuperada y reformulada por Kant en la obra de 1793 La religión dentro de los límites de la mera razón (Religion), bajo la denominación «esquematismo de la determinación del objeto» (Schematism der Objektbestimmung) y «esquematismo de la analogía» (Schematism der Analogie), respectivamente; cfr. Religion (Ak, VI, 65 Anm.).

$17 \mathrm{La}$ dualidad intuición/concepto es el reflejo epistemológico de la dualidad entre el caso particular (token) ubicado espaciotemporalmente y la clase general o tipo (type); Cfr. Strawson, P., op.cit., p. 22. 
ámbito teórico: conceptos empíricos, conceptos sensibles puros (o de la matemática en general) y conceptos a priori o categorías.

\section{La cuestión del significado en el dominio teórico: conceptos empíricos y sensibles puros}

Los tres momentos por los que llevamos a unidad cualquier diversidad dada -esto es, independientemente de la naturaleza de las representaciones que entran en juego- se concretan en: a) la comparación (Komparation) de una pluralidad de representaciones, atendiendo a sus posibles semejanzas/diferencias; b) la reflexión (Reflexion), que aísla aquello que es común a una diversidad y lo fija en una representación singular; c) la abstracción (Abstraktion), que prescinde de los elementos en los que difieren las distintas representaciones para centrarse en aquello que es idéntico (en lo semejante) a todas ellas 18 .

Así, en el caso de nuestros conceptos empíricos, es a partir de la comparación de un abeto, un sauce y un tilo, que podemos percibir las diferencias que median en relación a la forma, tamaño y color de sus troncos, ramas y hojas. Mas, si reflexionamos sobre aquellos aspectos que son comunes, observamos que el tronco, las hojas y las ramas mismas son elementos que comparten los tres, de tal modo que si prescindimos o abstraemos de las diferencias en forma, tamaño y color generamos el concepto de «árbol» 19 . Es precisamente por medio del concepto, en cuanto representación de una unidad que es válida para una diversidad de representaciones, que determinamos como «esto» o «aquello» la mera pluralidad indiferenciada de aquello que se nos presenta en la intuición. En este sentido, cabe afirmar que "el entendimiento es, en su acepción más general, la capacidad de deslinde [Vermögen der Grenzsetzung] pura y simplemente: es lo que "hace detenerse" [«zum Stehen bringt»] a la misma acción continua de las representaciones y lo que ayuda a trazarse una determinada imagen" 20 . La posibilidad del conocimiento consiste pues en último término en la posibilidad de «determinar» la pluralidad dada en la intuición, un deslinde por el cual aquello que se nos aparece lo hace siempre con una imagen determinada.

\footnotetext{
18 Cfr. Logik, §6 (Ak, IX, 94).

19 Íbid. Un ejemplo análogo de este proceso por el que obtenemos representaciones unitarias que valen para una diversidad de objetos lo encontramos en la descripción kantiana de la formación de una «idea normal estética»; cfr. KU, $\$ 17$ (Ak, V, 231 ff). Sobre este punto, cfr. Düssing, K., op.cit., p. 52; Lohmar, D., "Husserl's Type and Kant's Schemata. Systematic Reasons for Their Correlation or Identity", op.cit., p. 104.

20 Cassirer, E., Kant. Vida y doctrina, México, FCE, 1993, p. 368.
} 
De acuerdo con esta descripción, cabe afirmar que toda representación conceptual contiene un conjunto de rasgos o características (Merkmal) que pueden en principio ser compartidos por una diversidad de representaciones ${ }^{21}$. La plétora de características que están contenidas en un concepto y por las que nos es en general posible reconocer un particular como siendo subsumible bajo él constituyen el contenido (Inhalt) o «intensión» del concepto. Por otro lado, el conjunto de los objetos que pueden efectivamente ser subsumidos bajo un determinado concepto conforma su «extensión» (Umfang)22. Con las matizaciones que señalaremos más adelante, es precisamente la intensión de un concepto, el conjunto de las características que lo definen, aquello que constituye el fundamento de la relación particular-tipo.

Para Kant, toda característica presenta una doble función ${ }^{23}$ :

a) en la medida en que es una parte del conjunto de los rasgos que conforman la representación global del objeto, toda característica constituye una «representación parcial» (Partialvorstellung) del mismo que actúa como «fundamento de conocimiento» (Erkenntnisgrund) del objeto en su totalidad24;

b) toda característica proporciona asimismo un criterio de aplicación del concepto, i.e. aquel rasgo cuyo cumplimiento por parte del objeto lo hace susceptible de ser subsumido bajo el concepto en cuestión. Es precisamente el conjunto de estas características el que establece las condiciones de aplicación de nuestros conceptos, si bien cabe afirmar que la capacidad de aplicar o reconocer tales características en el objeto -la facultad de juzgar en cuanto capacidad de dirimir si una diversidad es o no subsumible bajo una regla de unificación dada, constituye un talento natural que no está sometido a reglas y que, por este motivo, puede ejercerse, ejercitarse mas no enseñarse 25 .

En la intensión de un concepto encontramos pues características que son parte de la representación global del objeto y cuyo cumplimiento por parte de aquello que se nos presenta lo hace susceptible de ser subsumido bajo tal concepto. La suma de estos rasgos delimita cierto «margen de maniobra» (Spielraum) formado por aquellas características que son esenciales a toda posible concreción del concepto, así

\footnotetext{
21 Con respecto a la poco trabajada «teoría de las características» kantiana, uno de los escasos textos en que Kant trata de manera explícita la cuestión es: Logik, VIII (Ak, IX, 58ff).

22 Cfr. Logik, §7 (Ak, IX, 95).

${ }^{23}$ Cfr. Hanna, R., "Kant's Theory of Empirical Judgment and Modern Semantics", op.cit., p. 342.

24 "Una característica es aquello en una cosa que constituye una parte del conocimiento de la misma; o, lo que es lo mismo, una representación parcial, en la medida en que es considerada como fundamento de conocimiento de la representación en su totalidad"; Logik, VIII (Ak, IX, 58). La utilización del término «cosa» (Ding) en este contexto podría explicarse por el hecho de que en la Lógica de Jäsche encontramos una compilación de apuntes de clase que se remontan al período precrítico kantiano.

25 Cfr. KrV, B171ff/A132ff.
} 
como posibles variaciones (en posición, perspectiva, tamaño o forma) con respecto a estas características que determinan un ámbito de expectativas posibles en relación a ese objeto ${ }^{26}$. En el caso de nuestros conceptos empíricos, estas expectativas dependerán de nuestra experiencia previa; así, la continua experiencia de nuevos objetos que son efectivamente subsumibles bajo un concepto determinado, nos permite ampliar el horizonte de expectativas mediante la admisión de nuevos rasgos 27 .

De este modo, en la intensión de nuestros conceptos empíricos y sensibles puros encontramos aquellas características que deben poder hallarse en toda posible concreción del concepto junto a posibles variaciones en el objeto 28 . Mas, dado que estas representaciones conceptuales deben valer para una diversidad de objetos que, a pesar de presentar características comunes, difieren ciertamente en muchos otros aspectos, su intensión no podrá incluir todas las características que pueda presentar cualquier imagen del objeto ${ }^{29}$, ya que el particular satisface tan sólo una esfera de la universalidad pensada en el concepto. En este sentido afirma Kant que el concepto empírico de un perro significa "una regla según la cual mi imaginación puede esbozar [verzeichnen] de manera general la forma [Gestalt] de un animal cuadrú-

\footnotetext{
26 Es decir: "Los objetos pueden cambiar, pero no pueden cambiar, por así decir, al margen de todo reconocimiento. Si así sucediera, no podríamos saber que habían cambiado, pues no podríamos reconocerlos en cuanto cambiados (...). Es decir, nuestros conceptos de los objetos y los criterios de reidentificación que encarnan, deben considerar cambios del mundo objetivo sujetos a la limitación de que el cambio debe ser coherente con la posibilidad de aplicar esos conceptos y criterios en la experiencia. ¿Cómo se satisface este requisito? (...) nuestros conceptos de los objetos están enlazados con conjuntos de expectativas condicionales acerca de las cosas que percibimos subsumidas en ellos"; Strawson, P., op.cit., p. 127ss.

27 Como veremos, una de las funciones de la imaginación consiste en variar la imagen esperada del objeto de acuerdo con el esquema del concepto, de tal modo que la representación conceptual pueda aplicarse a particulares que no se ajusten enteramente a las condiciones especificadas por el concepto; cfr. Lohmar, D., "Husserl's Type and Kant's Schemata", op.cit., p. 110.

28 Si bien es cierto que los conceptos sensibles puros, por no depender de la experiencia, no están sujetos al mismo nivel de variación que los conceptos empíricos, es imposible que la matemática pura llegue a un conocimiento íntegro de su objeto: "ya que aquélla [la metafísica], puesto que el objeto en ella debe ser siempre comparado con todas las leyes necesarias del pensamiento, debe dar un número determinado de conocimientos que se dejan agotar del todo. Éstas, sin embargo, dado que ofrecen una diversidad infinita de intuiciones (puras o empíricas), objetos del pensamiento por tanto, no logran nunca una completud absoluta, sino que pueden ser extendidas hacia el infinito, como la matemática pura y la doctrina empírica de la naturaleza"; Metaphysische Anfangsgründe der Naturwissenchaft (Ak, IV, 473).

29 "¿Cómo entender que un algo no dado se represente a nuestra conciencia como si se tratase de una cosa dada? En esta pregunta se cifra todo el "misterio" psicológico de la formación de los conceptos. Hay que poner de manifiesto cómo una intuición sensible, que, en cuanto tal, sólo puede señalar un determinado contenido concreto, puede llegar a adquirir la capacidad necesaria para convertirse en expresión de una clase total de contenidos y para reproducir cada uno de éstos en su estructura constitutiva"; Cassirer, E., El problema del conocimiento en la filosofía y la ciencia modernas, vol. II, México, FCE, 1956, p. 665.
} 
pedo, sin estar limitada a ninguna forma particular que la experiencia me ofrece, o a cualquier posible imagen que pueda presentar en concreto"30. Por este motivo, nuestros términos lingüísticos no significan la imagen sino "la regla según la cual mi imaginación puede esbozar [ver-zeichnen] de manera general" cualquier imagen particular del concepto en cuestión ${ }^{31}$.

La representación de este "modo general de proceder de la imaginación para proporcionar a un concepto su imagen" es lo que constituye "el esquema [Schema] en relación a ese concepto"32. El esquema, «mistérica» producción del alma humana $^{33}$, regula el campo de sensibilizaciones posibles de un concepto. A diferencia de la imagen, aquello que hallamos en el esquema es un «esbozo» (Skizze) del objeto, i.e. una representación del objeto que si bien no puede atender a sus contornos precisos es suficiente para reconocer cualquier particular como siendo subsumible bajo ese concepto. $\mathrm{O}$, dicho a la inversa: los esquemas constituyen las «condiciones de aplicación» (Anwendungsbedingungen) de nuestros conceptos 34 .

El fundamento de la relación particular-tipo en el dominio teórico es el esquema en cuanto modo general de proceder por el que la imaginación puede proporcionar una imagen a un determinado concepto. Así, el esquema constituye la intensión de todo concepto empírico o sensible puro, siendo en último término la imagen del objeto producida sintéticamente a partir del material sensible dado el elemento particular en la relación de hipotiposis ${ }^{35}$.

\section{Crítica a la concepción del conocimiento como copia}

Como hemos desarrollado en el apartado precedente, el contenido de nuestros conceptos empíricos y sensibles puros es el esquema en cuanto regla por la que

$30 \mathrm{KrV}, \mathrm{A} 141 / \mathrm{B} 180$.

31 Ibid. Precisamente porque la intensión de nuestros conceptos empíricos y sensibles puros no contiene la totalidad de características que son propias de un particular determinado, el esquema no proporciona una descripción completa del objeto. $\mathrm{Y}$ es en este sentido que forzamos la traducción del verbo «verzeichnen» (indicar, registrar), resaltando el carácter «defectuoso» de la acción indicado por la partícula alemana «ver-».

32 Íbid.

33 "Este esquematismo de nuestro entendimiento, en vistas de los fenómenos y su mera forma, es un arte oculto en la profundidad del alma humana, cuyo verdadero manejo difícilmente podrá ser arrancado a la naturaleza y descubierto ante los ojos"; KrV, A141/B180.

34 Esta es la tesis principal de los artículos ya citados de R. E. Butts y de D. Lohmar.

35 "La expresión utilizada por los lógicos antiguos y nuevos, un objeto está «contenido bajo un concepto» es imposible hasta que uno no deviene consciente de que por «objeto» no debe entenderse una cosa sino siempre la imagen de una cosa, y que el discurso en torno al «estar-contenido» en el sentido de un tránsito del concepto universal a la imagen debe ser comprendido por medio del esquema"; Kaulbach, F., „Schema, Bild und Modell nach den Voraussetzungen des Kantischen Denkens“, Studium Generale 18 (1965), p. 460. 
puede esbozarse una imagen particular del concepto en cuestión. Aquello que nos presenta el esquema de un concepto es un conjunto de características que, si bien no abarcan la suma de todas las posibles variaciones del objeto, establece las condiciones cuyo cumplimiento por parte del objeto nos permite aplicar ese concepto.

Mas, en cuanto representación de un conjunto de rasgos que son compartidos por una diversidad de objetos, todo concepto supone la efectiva estructuración de aquello que se nos presenta. Es decir: entender el significado en términos de rasgos o características no implica una concepción del conocimiento como «copia», i.e. una mera trasposición mental de las características de los objetos «externos», puesto que todo concepto se funda en el acto por el que introducimos un orden en el enlace de la diversidad de nuestras representaciones, ya sean empíricas o puras.

El concepto, en cuanto representación de una unidad que es válida para una diversidad de particulares, no es algo inmediatamente dado en la intuición, sino que resulta de la actividad espontánea (Selbsttätigkeit) del sujeto 36 . Porque la diversidad dada ha sido aprehendida, recorrida y enlazada de una determinada manera es en general posible llegar a la conciencia de una representación cuyas características son compartidas por una diversidad (conceptus communis) ${ }^{37}$. Y es en este sentido que debe entenderse la caracterización kantiana del significado en términos esencialmente activos, a saber, aquella "regla por la que nuestra imaginación puede esbozar la imagen de un objeto", en oposición a la reflexión sobre la naturaleza de nuestros conceptos ofrecida por Locke, Berkeley y Hume.

Aun cuando no siempre tenida suficientemente en cuenta, es de fundamental importancia la referencia al contexto histórico-dialógico empirista para ubicar la teoría del esquematismo en Kant ${ }^{38}$. En la medida en que toda representación conceptual -en términos de Locke, toda «idea» asociada a un término general- debe

\footnotetext{
36 Cfr. $\mathrm{KrV}, \mathrm{B} 130$.

37 Cfr. KrV, B133-134.

38 Cfr. Philonenko, A., "Lecture du schématisme transcendantal", en Philonenko, A., Études kantiennes, Paris, J. Vrin, 1982, §§2-3; Düssing, K., op.cit., pp. 56-61. Para Philonenko, el apartado del «Esquematismo Trascendental» sirve de «contraprueba» a la Deducción Trascendental de las Categorías. En el capítulo del Esquematismo, Kant plantea el problema de la aplicación de lo universal a lo particular en los términos psicológicos propios del empirismo (y, en especial, de Berkeley) para mostrar su esencial ineficacia, lo cual funciona como plataforma para la reelaboración en 1787 de la Deducción Trascendental de las Categorías desde un punto de vista más lógico y menos psicológico que en su primera versión. Precisamente porque esta contraprueba es eficaz, i.e. porque en el Esquematismo Trascendental se muestra la imposibilidad de una deducción empírica de las categorías, este capítulo constituye el único no modificado en la segunda edición de $\mathrm{KrV}$, un hecho que sirve para desmentir la tesis de M. Heidegger conforme a la cual en la segunda edición se produce un retroceso por parte de Kant frente a la imaginación trascendental en cuanto fundamento último de la posibilidad del conocimiento. Si así fuera, no tendría sentido que el único capítulo que permanece inalterado en 1787 fuera precisamente aquél en que se trata específicamente de la imaginación; cfr. Philonenko, A., op.cit., $§ 4$.
} 
valer para una diversidad de objetos que, a pesar de tener características comunes, pueden diferir en otros muchos aspectos, nos topamos con la paradoja de que conforme a la exigencia de validez universal el significado asociado al término general «triángulo» debería consistir en la representación de un triángulo tal que no podría ser ni equilátero, ni isósceles ni escaleno sino "todos y ninguno al mismo tiempo"39. Es en este sentido que para Locke el significado de nuestros términos generales lo constituye una «idea general abstracta ${ }^{40}$.

Mas, como afirma Berkeley, nuestra mente trata siempre con ideas particulares, es decir, con imágenes concretas de los objetos, de tal modo que cuando nos preguntamos por el significado de un término general cualquiera no podemos dejar de asociar una imagen determinada del objeto. No es cierto por tanto que el significado de nuestros términos pueda identificarse con una representación general de carácter indeterminado (una idea general abstracta en el sentido de Locke), esto es, la representación de un triángulo que no sea ni equilátero, ni escaleno, ni isósceles ${ }^{41}$. Ahora bien, si tratamos siempre con ideas particulares: ¿cómo es posible justificar que aquello que demostramos con respecto a un triángulo particular haya de ser válido para todo triángulo?

Según Berkeley, la validez universal de una demostración matemática se funda en que en ella prescindimos de los aspectos que son particulares a la figura sobre la que se está efectuando la demostración, i.e. la magnitud de sus ángulos, la longitud de sus lados, etc.42. Mas, ¿qué diferencia hay entre prescindir de aquello que es exclusivo y particular de la figura -podríamos decir, centrarse en aquello que es esencial a todo triángulo, en el carácter triangular de la figura misma- y considerar que el término «triángulo» significa aquello que es común a todo triángulo? Berkeley no niega la existencia de ideas generales, sino el que podamos formarnos

39 “... la idea general de triángulo (...). Pues no ha de ser la idea de un triángulo equilátero, ni isósceles, ni escaleno, sino la de todos y ninguno al mismo tiempo. En efecto, se trata de algo imperfecto que no puede existir, una idea en la que algunas ideas diferentes e incompatibles entre sí, se ponen juntas. Verdad es que la mente, en este imperfecto estado suyo, necesita de ideas así, y recurre a ellas en cuanto puede por conveniencias de comunicación y para aumentar el conocimiento, cosas ambas a las que naturalmente se ve inclinada"; Locke, J., Ensayo sobre el entendimiento humano, México, FCE, 1956, Libro IV, cap. 7, §9, p. 597.

40 Con respecto a la concepción semántica de Locke, que se corresponde a la pregunta por el significado de nuestros términos generales, puede consultarse: Mackie, J. L., Problemas en torno a Locke, UNAM, México, 1988, §4; García-Carpintero, M., Las palabras, las ideas y las cosas, Ariel, Barcelona, 1996, $\$ 4$.

41 “... un hombre podrá considerar una figura meramente como triangular, sin fijarse en las cualidades particulares de los ángulos o en las relaciones particulares entre los lados. Hasta aquí puede abstraer; pero esto no probará nunca que pueda formarse una inconsistente, abstracta idea general de triángulo"; Berkeley, G., Tratado sobre los principios del conocimiento humano, Madrid, ed. Gredos, 1982, Introducción, §16, p. 41.

42 Íbid. 
una representación general de carácter indeterminado (una idea general abstracta). La demostración se efectúa siempre sobre la imagen de un triángulo concreto, si bien las conclusiones de tal demostración tienen valor universal en la medida en que el triángulo que nos imaginamos "está en lugar de" y "representa" (stand for and represent) a todos los triángulos 43 .

Para expresarlo en los términos que utiliza Berkeley: la generalidad que otorgamos al triángulo sobre el que realizamos la demostración la adquiere porque tal idea particular actúa como un signo que representa a la totalidad de objetos de la misma clase ${ }^{44}$. La universalidad no consiste en la capacidad para formarse una idea indeterminada, sino en la relación entre tal representación concreta y los elementos de la misma clase a los que representa. Sucede algo análogo a lo que acontece con los objetos de la visión en relación a los del tacto: unas determinadas ideas particulares de la vista funcionan como el signo de aquellas otras ideas particulares del tacto que obtendríamos si realizásemos tales o cuales movimientos ${ }^{45}$.

De acuerdo con Hume, todo signo lingüístico detentaría para Berkeley una doble función $46:$ a) en primer lugar, representa efectivamente una imagen o idea particular; b) pero en virtud del hábito por el que hemos aprendido a aplicar un mismo signo a objetos semejantes (mas no completamente iguales), el sujeto le asocia una «zona de comprensión» suficientemente amplia como para que nos permita reconocer las diferencias entre objetos de una misma clase 47 . En este sentido afirma Berkeley que "una palabra deviene general cuando se hace de ella el signo, no

43 "la universalidad (...) no consiste en la absoluta, positiva naturaleza o concepción de algo, sino en la relación que se establece con los particulares a los que representa y está en lugar de"; Berkeley, G., op.cit., Introducción, §15, p. 40.

44 “... una idea que, considerada en sí misma, es particular, deviene general al hacer que represente o esté en lugar de todas las demás ideas particulares de la misma clase (...) dicha línea, que en sí misma es particular, es, sin embargo, general con respecto a lo que ella significa; pues, tal y como aquí es utilizada, representa todas las demás líneas particulares, cualesquiera que éstas sean (...) esa línea particular se convierte en general al hacerse de ella un signo"; Berkeley, G., op.cit., Introducción, §12, pp. 36-37.

45 "En general creo muy bien podemos concluir que los objetos propios de la visión constituyen un lenguaje universal del Autor de la naturaleza, mediante el cual se nos instruye acerca de cómo regir nuestras acciones, a fin de alcanzar aquellas cosas que son necesarias para la preservación y el bienestar de nuestros cuerpos, como también para evitar cualquier cosa que pueda dañar y destruir $(\ldots)$ la manera como significan y marcan en nosotros los objetos que están a la distancia, es igual que los lenguajes y signos de creación humana los que no sugieren las cosas significadas debido a ninguna similitud o identidad de naturaleza, sino sólo por una conexión habitual que hemos observado entre ellos"; Berkeley, G., Ensayo sobre una nueva teoría de la visión, Buenos Aires, Aguilar, 1973, §147, p. 119.

46 Cfr. Robles, J. A., “Abstracción y generalidad” en Estudios Berkeleyanos, México, UNAM, 1990, pp. 77-99.

47 Cfr. Hume, D., Tratado de la naturaleza humana, Madrid, Tecnos, 1988, Parte I, Libro I, Sección VII, pp. 62-72. 
de una idea general abstracta, sino de varias ideas particulares, cualquiera de las cuales, indiferentemente, es sugerida a la mente por dicho signo" 48 . De este modo, la validez universal de nuestro conocimiento se funda en último término en la experiencia, esto es, en esa zona de comprensión generada a partir de la aplicación de un mismo signo a particulares diferentes que son reconocidos no obstante como perteneciendo a una misma clase.

Por todo lo dicho, resulta claro que el empleo por parte de Kant del ejemplo del concepto de triángulo en el apartado del «Esquematismo Trascendental»49 es una referencia implícita a la discusión generada a partir de la pregunta por el significado de nuestros términos generales planteada por J. Locke en el Ensayo sobre el entendimiento humano. Tal y como Kant la recibe, la pregunta por el significado de nuestros términos generales debe tener en cuenta los siguientes dos aspectos:

a) el hecho de que nuestros conceptos hayan de valer para una diversidad de objetos que, a pesar de compartir ciertas características, difieren en muchas otras, implica que el conjunto de rasgos que conforman su intensión no puede circunscribirse a las características que pueda presentar cualquier imagen particular del objeto sino que, como sostiene Locke, debe poder representar a "todos y ninguno" a la vez. En este sentido afirma Kant que "al concepto de un triángulo en general jamás le sería adecuada ninguna imagen del mismo. Ya que la imagen no alcanzaría la universalidad del concepto, que hace que el concepto sea válido para todos los triángulos, ya sean rectángulos u oblicuos, sino que estaría siempre limitado tan sólo a una parte de esta esfera" 50 ;

b) ahora bien, como afirma Berkeley, esto no implica que se asocie una imagen indeterminada a nuestros conceptos. Siempre tratamos con una imagen particular del objeto, ya sea porque todo aquello que puede en general presentársenos lo hace con una determinada imagen, ya porque la imaginación reproductiva asocia siempre una imagen concreta a todo signo lingüístico.

Así, si bien es cierto que nuestra mente se ocupa con imágenes particulares de los objetos, la intensión de nuestros conceptos empíricos o sensibles puros no puede identificarse con ninguna imagen concreta que podamos representarnos. En este sentido afirma Kant que "en la base de nuestros conceptos sensibles puros [i.e. los conceptos de la matemática en general] no yacen imágenes sino esquemas de los objetos" 51 . El concepto de un triángulo significa aquel modo general en que se lleva a cabo el proceso de recorrer, enlazar y aprehender en una unidad (la figura geo-

\footnotetext{
48 Berkeley, G., Tratado sobre los principios del conocimiento humano, op.cit., Introducción, §11, p. 35.

49 Cfr. KrV, B180/A141.

50 Íbid.

$51 \mathrm{KrV}, \mathrm{B} 180 / \mathrm{A} 140$.
} 
métrica) la diversidad (de puntos) en el espacio, a saber, por medio del trazo (la construcción) de tres rectas que se cortan mutuamente en un solo punto. Este procedimiento, por medio del cual construimos una figura geométrica de tres ángulos, es válido para todo triángulo independientemente del aspecto concreto que pueda adoptar.

Asimismo, cinco puntos colocados consecutivamente «" ${ }^{\cdots}$ " constituyen una imagen del número cinco; mas, pensar el concepto de número es pensar una sucesiva adición de unidades de las que cabe suponer la homogeneidad para que sea posible su aditamento. El concepto de número consiste en aquel procedimiento general por el que subsumimos la diversidad pura (el tiempo entendido como la sucesión de instantes, el mero «uno-tras-otro») bajo la categoría de cantidad (una pluralidad de elementos que llevada a unidad nos proporciona una totalidad) ${ }^{52}$.

La pregunta por el significado de nuestros términos implica en Kant una crítica a la concepción lockeana del conocimiento como copia, i.e. la trasposición de las características de los objetos externos en la mente del sujeto, que reposa en último término sobre una concepción metafísica substancialista del conocimiento de cuño cartesiano. La anulación de este modelo pasa por el reconocimiento del carácter esencialmente constitutivo del sujeto en la conformación como objeto de aquello que se nos presenta en la intuición, un reconocimiento que Kant recoge en su definición del esquema como procedimiento y que excluye toda consideración de la relación particular-tipo en términos de similitud.

\section{La cuestión del significado en el dominio teórico: los conceptos a priori del entendimiento}

Para el ser racional finito, la posibilidad del conocimiento consiste en la posibilidad de una estructuración unitaria de aquello que se le presenta en la intuición. El conocimiento de un objeto conlleva que el enlace establecido entre una diversidad de representaciones no acontece aleatoriamente sino que tiene lugar de acuerdo con una regla que introduce cierto orden en la sucesión de las mismas ${ }^{53}$. En cuanto

\footnotetext{
52 "El contenido semántico del concepto de número no es caracterizado extensionalmente, en términos de un dominio de entidades, sino intensionalmente, en términos de una clase de procedimientos gobernados por una regla"; Wong, W-CH., "On a Semantic Interpretation of Kant's Concept of Number", Synthese, vol. 21, Number 3, December 1999, p. 357. Con respecto a la semántica de nuestros conceptos sensibles puros, cfr. Hanna, R., "Kant, Truth and Human Nature", British Journal for the History of Philosophy 8(2), Routledge, 2000, pp. 225-250.

53 "Si el cinabrio fuera ahora rojo, ahora negro, ahora ligero o pesado (...) mi imaginación empírica ni siquiera dispondría de la ocasión de tener en el pensamiento el pesado cinabrio con la representación del color rojo (...); sin que aquí dominara una cierta regla a que los fenómenos estuvieran sometidos por ellos mismos, no podría haber ninguna síntesis empírica de reproducción”; KrV, A100-101.
} 
representación de una regla de unificación de lo diverso, todo concepto se basa en la unidad de acción por medio de la cual se subsumen diversas representaciones bajo una representación común 54 .

Ahora bien, la posibilidad de la acción misma de recorrer, enlazar y aprehender unitariamente la diversidad de lo dado requiere una visión previa de los distintos modos posibles de unión, esto es, de cada uno de los modos en que es en general posible para el entendimiento pensar la unidad en la diversidad (cantidad, calidad, relación y modalidad) y que constituye lo que Kant denomina «concepto a priori del entendimiento»o «categoría», i.e. "representaciones que pertenecen de antemano a la estructura esencial de la reflexión, es decir, que actúan en, con y por la reflexión, siendo en suma conceptos reflexionantes (reflektierende Begriffe)"55.

Con respecto a los conceptos a priori del entendimiento, y a diferencia de los conceptos empíricos y sensibles puros, no es posible proporcionar una imagen que les corresponda puesto que constituyen aquellas condiciones que están involucradas en toda estructuración unitaria de aquello que se nos presenta, i.e. en toda generación de reglas ${ }^{56}$. Es decir: el proceso por el que la imaginación es capaz de proporcionar una imagen que corresponda al concepto de un determinado concepto (empírico o sensible puro) se articula según las funciones de unidad de nuestro pensamiento, y es por este motivo que los conceptos a priori del entendimiento son aquellas reglas involucradas en toda posible generación de reglas 57 .

La posibilidad de una estructuración unitaria de aquello que se nos presenta en la intuición tiene lugar de acuerdo con los diversos modos en que nos es en general posible llevar a unidad la diversidad de aquello que se nos aparece. En este sentido, la posibilidad del conocimiento para el ser racional finito no sólo supone: a) la necesaria referencia a aquello que se nos presenta en la intuición y, por tanto, el tiempo en cuanto forma a priori originaria de la sensibilidad; b) sino la capacidad de conservar y volver a presentar de un modo determinado el conjunto de las sucesivas aprehensiones de aquello que se nos aparece.

\footnotetext{
54 "Todas las intuiciones, en cuanto sensibles, se basan en afecciones; los conceptos, por tanto, en funciones. Por función entiendo la unidad de la acción por la que se ordenan diferentes representaciones bajo una de común"; KrV, A68/B93.

55 Heidegger, M., Kant y el problema de la metafísica, México, FCE, 1986, p. 54.

56 "Por el contrario, el esquema de un concepto puro del entendimiento es algo que no puede ser llevado a ninguna imagen, sino que es tan sólo la síntesis pura, conforme a una regla de la unidad según conceptos en general"; KrV, B181/A142.

57 "La filosofía trascendental tiene empero la especificidad de que, además de la regla (o, mejor, la condición universal para regular) que es dada en el concepto puro del entendimiento, al mismo tiempo debe mostrar a priori el caso en el que ha de ser aplicado"; KrV, B174/A135 (la negrita es nuestra). En cuanto condiciones envueltas necesariamente en toda posible generación de reglas, los conceptos a priori del entendimiento constituirían reglas de segundo orden.
} 
La posibilidad del conocimiento es pues la posibilidad de una determinación objetiva de la sucesión de representaciones en nuestro sentido interno, y los distintos modos en que es en general posible determinar objetivamente el tiempo en cuanto modo en que se nos presenta todo aquello que en cada caso puede presentársenos conforman las características trascendentales de todo objeto posible, i.e. aquellas condiciones por las que es posible nuestro conocimiento y que constituyen al mismo tiempo las determinaciones a priori de todo objeto posible 58 . De la aplicación de las categorías a la forma a priori originaria de la sensibilidad resultan los esquemas trascendentales de la imaginación en cuanto determinaciones trascendentales del tiempo: la sucesión en el tiempo (Zeitreihe), su contenido (Zeitinhalt), el orden (Zeitordnung) y el tiempo en su totalidad (Zeitinbegriff) $)^{59}$.

$\mathrm{Y}$ es en este contexto donde cobra pleno sentido la noción de exposición esquemática en cuanto modelo de significación operante en el dominio teórico: la sensibilización de nuestros conceptos empíricos y sensibles puros tiene lugar mediante un esquema, no sólo porque toda relación particular-tipo acontece gracias al esquema que corresponde a ese concepto, sino porque la relación misma de hipotiposis se articula en último término desde los esquemas trascendentales de la imaginación en

\section{$58 \mathrm{Cfr} . \mathrm{KrV}, \mathrm{B} 182 \mathrm{ff} / \mathrm{A} 142 \mathrm{ff}$.}

59 Cfr. KrV, B177/A138. El objetivo de la «Deducción Trascendental de las Categorías» en sus dos ediciones (KrV, B129ff/A96ff) es justificar la posibilidad de una aplicación de los conceptos a priori del entendimiento a los fenómenos. Y tiene sentido preguntarse por la legitimidad de la aplicación de las distintas funciones de unidad del pensamiento a aquello que se nos presenta en la intuición, como consecuencia de la naturaleza estrictamente heterogénea de ambos elementos; a saber, el carácter universal y necesario de las categorías en cuanto formas a priori del pensamiento por un lado y la estricta singularidad que es propia del material sensible por otro.

La justificación de la validez objetiva de las categorías consiste pues en la mostración de su posible aplicación a la intuición en general. El núcleo del argumento se condensa en la afirmación kantiana de que nuestro conocimiento se ocupa fundamentalmente con los fenómenos de las cosas y no con aquello que las cosas son en sí mismas. Todo aquello que es tiene sentido en relación a un sujeto y, por tanto, de acuerdo con las condiciones que hacen posible tal relación; así, la acción sintética del yo está presente ya en el nivel más originario de la donación, de tal modo que puede afirmarse que el conjunto de las condiciones que hacen posible un conocimiento del objeto son las mismas por las que es en general posible que haya algo que pueda constituirse como objeto, dándose por tanto una identificación de fondo entre la apercepción trascendental y el objeto trascendental en relación a su forma (cfr. $\mathrm{KrV}, \mathrm{A} 109)$.

Una vez que ha quedado establecido que la aplicación de las categorías a la intuición en general es posible cabe plantearse cómo es posible. La pregunta por la efectiva aplicación de las categorías implica la restricción del ámbito de aplicación a nuestra forma específica de intuición. Si en la Deducción Trascendental ha quedado establecido que es por medio de su posible referencia a la intuición en general que las categorías adquieren realidad objetiva, la pregunta por su efectiva aplicación reviste en el «Esquematismo Trascendental» y la «Analítica de los Principios» la forma de una determinación trascendental del tiempo en cuanto condición inexcusable del conocimiento para todo ser racional finito; cfr. Detel, W., „Zur Funktion des Schematismuskapitels in Kants Kritik der reinen Vernunft“, KantStudien, 69 (1978). 
cuanto determinaciones trascendentales del tiempo, y de ahí la caracterización de la exposición esquemática como algo que tiene lugar "cuando a un concepto que es aprehendido por el entendimiento le es dada la correspondiente intuición a priori" 60 .

\section{El concepto de «exposición simbólica»: los conceptos de razón}

La cuestión del significado en el dominio teórico equivale a la pregunta por el fundamento de la relación entre el concepto y la intuición del objeto. La posibilidad de referir un concepto a su objeto es aquello en que consiste precisamente la validez objetiva del mismo, lo que supone en último término su conformidad con los principios trascendentales del entendimiento en cuanto condiciones a priori de la posibilidad de la experiencia61. Así, la mostración de un ejemplo del mismo asegura que un concepto empírico es objetivamente válido; por otro lado, los conceptos de la matemática en general o conceptos sensibles puros dependen de su construibilidad en la intuición pura, en el espacio para los conceptos de la geometría y en el tiempo para los conceptos de la aritmética ${ }^{62}$.

Porque las ideas de la razón -Dios, alma, libertad- no se ajustan a las condiciones a priori de la experiencia carecen de un significado cognoscitivamente válido63. Es precisamente la reflexión sobre los límites en los que debe inscribirse todo uso legítimo de nuestras facultades cognoscitivas, aquella cuestión que no llega a plantearse nunca el filósofo dogmático ${ }^{64}$. En este sentido, la «crítica» de la «razón pura» puede entenderse como la necesaria «censura» a que debe someterse a la razón que opera desde, en y por ella misma, es decir, en todo uso de nuestros conceptos que prescinda de las demás facultades cognoscitivas y, en particular, de la sensibilidad65. Sin esta labor crítica previa, la metafísica queda reducida a un mero

$60 \mathrm{KU}, \S 59(\mathrm{Ak}, \mathrm{V}, 351)$.

$61 \mathrm{cfr} . \mathrm{KrV}, \mathrm{B} 198 \mathrm{ff} / \mathrm{A} 159 \mathrm{ff}$.

62 "Cuando a un concepto puede serle dada su correspondiente intuición a priori, se dice que este concepto ha sido construido. Si se trata tan sólo de una intuición empírica, se le denomina un mero ejemplo de este concepto. La acción de añadir la intuición al concepto se dice en los dos casos exposición (exhibitio) del objeto, sin la cual (puede darse de manera directa o indirecta) no puede haber ningún conocimiento"; Fortschritte (Ak, XX, 325).

${ }^{63}$ Suscribimos pues la semántica verificacionista propuesta por Strawson, mediante el establecimiento del «Principio de Significatividad», como clave de lectura de la filosofía teórica de Kant; cfr. nota 5 del presente escrito.

64 "El dogmatismo es, por tanto, el proceder dogmático de la razón pura sin crítica previa de su propia capacidad"; KrV, BXXXV.

65 "Vernunft tiene muchos significados, en Kant. Sensu lato (y casi identificándose con el entendimiento) es la «facultad superior de conocimiento» (frente a la inferior: la sensibilidad); cfr. $\mathrm{KrV}$, A835/B863. Este es uno de los sentidos del título de la obra: Crítica de la razón pura equivale a «censura de la razón desligada de la sensibilidad», como vio muy bien C. Ch. E. Schmid en su Programa 
juego de representaciones, i.e. un encadenamiento de conceptos vacíos de un contenido cognoscitivamente válido según el principio de no contradicción66.

Ahora bien, que no es posible conocimiento alguno de estos conceptos de razón no implica que carezcan de contenido. La posibilidad de una estructuración unitaria de aquello que se nos presenta en la intuición se articula conforme a los conceptos a priori del entendimiento, de tal modo que aun cuando se suprima su contenido sensible toda representación conceptual conserva el pensamiento de una unidad en la diversidad67. Que las formas a priori de nuestro pensamiento no dependen del material sensible dado implica que los límites de lo real no son coextensivos con los límites espaciotemporales de nuestra experiencia ${ }^{68} \mathrm{y}$, en consecuencia, que existen ámbitos de validez allende el dominio teórico.

Concebida como la capacidad para determinarse a obrar con independencia de los estímulos sensibles, la idea de la libertad se define por su carácter no-investigable desde los parámetros del mundo natural 69 . La realidad objetiva de esta idea reside en la posibilidad de pensar sin contradicción en un mismo suceso la ley según la cual todo aquello ubicable espacio-temporalmente depende de la existencia de otro (causa) en su llegar a ser (efecto) y la capacidad espontánea de producir una serie de acontecimientos ${ }^{70}$. En cuanto fundamento del campo de lo suprasensible 71 , la libertad no es susceptible pues de una exposición directa en la intuición. Aún así, la experiencia de la belleza nos proporciona cierto correlato sensible de esta idea, sin que esta exposición detente empero valor constitutivo sino meramente explicativo ${ }^{72}$.

En este contexto se ubica el concepto de exposición simbólica, que se da cuando "a un concepto que sólo la razón puede pensar y al que ninguna intuición sensible puede serle adecuada, se le subsume una intuición con respecto a la cual el pro-

para el Semestre de Invierno de 1785/86, en el que ofrece lecciones sobre «la doctrina filosófica denominada Censura (Zensur) de la razón innata, en alemán (...) Critik der reinen Vernunft»”; Duque, F., Historia de la filosofía moderna, ed Akal, Madrid, 1998, p. 58ss.

66 "Si un conocimiento debe tener realidad objetiva, i.e. referirse a un objeto y tener en él significado (Bedeutung) y sentido (Sinn), el objeto debe poder ser dado de alguna manera. Sin esto son los conceptos vacíos, y por medio de ellos se ha pensado ciertamente algo, pero de hecho no se ha conocido nada mediante este pensamiento sino que tan sólo se ha jugado con representaciones"; KrV, B194195/A155.

67 "De hecho, en los conceptos puros del entendimiento permanece, también tras la separación de toda condición sensible, un significado lógico de la mera unidad de las representaciones"; KrV, B186/A147.

68 Cfr. Strawson, P., op.cit., p. 237.

69 Cfr. KU, Allg. Anmerk. zur Expox. Ästh. reflek. Urteilsk. (Ak, V, 275).

70 Algo asegurado en la resolución de la tercera antinomia de la razón pura; cfr. KrV, B472ff/A444ff.

71 Cfr. KU, Einleitung, II (Ak, V, 174).

72 "Este es el esquematismo de la analogía (como aclaración), del que no podemos prescindir. Transformar éste en un esquematismo de la determinación del objeto (como ampliación de nuestro conocimiento) es antropomorfismo, el cual es en perspectiva moral (en la religión) de las más perjudiciales consecuencias"; Religion (Ak, VI, 65Anm). 
ceder de la facultad de juzgar coincide de un modo meramente analógico con aquello que observa cuando esquematiza, i.e. en la regla de tal proceder solamente, mas no en la intuición misma; coincide por tanto según la forma de la reflexión meramente, y no en lo que respecta al contenido"73.

En cuanto relación de «hipotiposis», toda exposición simbólica presenta las siguientes características:

a) el «tipo» es una idea o concepto de razón, i.e. una representación para la que no existe intuición sensible alguna (empírica o pura) que pueda subsumírsele directamente (mediante la mostración de un ejemplo o construyendo $a$ priori el concepto en la intuición);

b) la exposición simbólica es un modo intuitivo de representación, no discursivo; es decir, por exposición simbólica no entiende Kant la utilización de signos sensibles, i.e. meros «caracterismos» (Charakterismen) que no contienen nada que corresponda a la intuición del objeto y que, como es el caso de las palabras y los símbolos algebraicos, se emplean para expresar conceptos 74 ;

c) la exposición simbólica es un modo intuitivo indirecto de representación; que no es posible encontrar intuición alguna que sea plenamente adecuada a un concepto de razón significa que no puede mostrarse un ejemplo del mismo (concepto empírico) ni construirse a priori en la intuición (concepto sensible puro). Ahora bien, dado que el conjunto de los objetos del mundo sensible constituye el campo de aplicación tanto del dominio teórico como del prácti$\mathrm{co}^{75}$, el referente intuitivo en la exposición simbólica de un concepto de razón debe expresar de manera analógica aspectos de una representación que rehúye por principio toda posible sensibilización, es decir, mediante el reconocimiento de una igualdad en la relación entre dos pares de elementos heterogéneos entre sí76.

\footnotetext{
$73 \mathrm{KU}, \S 59$ (Ak, V, 351). Una presentación del concepto de exposición simbólica en el marco de una investigación más general acerca de los distintos modos de exposición en la filosofía kantiana se encuentra en Mumbrú, A., "Informe de la tesi doctoral: El problema de la sensibilització de l'àmbit de la moralitat a Kant", en Anuari de la Societat Catalana de Filosofia, Barcelona, IEC, número XXI, 2011, pp.147-165.

${ }^{74}$ Cfr. KU, $\$ 59$ (Ak, V, 351ff). cfr. Flach, W., „Zu Kants Lehre von der symbolischen Darstellung”, Kant-Studien 73 (1982), pp. 452-462.

75 "El entendimiento y la razón tienen, por tanto, dos legislaciones diferentes sobre uno y el mismo suelo de la experiencia, sin que a uno le esté permitido influir sobre el otro"; KU, Einleitung, II, (Ak, $\mathrm{V}, 174)$.

76 "En filosofía, las analogías significan algo muy diferente a aquello que representan en las matemáticas. En éstas son fórmulas que expresan la igualdad de dos relaciones entre magnitudes, y son siempre constitutivas, de tal modo que si son dados tres miembros de la proporción, también es dado el cuarto, i.e. puede ser construido. Ahora bien, en filosofía la analogía no es la igualdad de dos relaciones cuantitativas sino cualitativas, donde a partir de los tres miembros dados tan sólo puedo conocer y dar a priori la relación con un cuarto, y no este cuarto miembro mismo, si bien tengo una regla para buscarlo en la experiencia y un rasgo para descubrirlo en ella"; KrV, B222/A179.
} 
Así, por ejemplo, el funcionamiento mecánico de un molino de viento y todas las características que podemos obtener de su concepto (automatismo, unidireccionalidad, rigidez, monotonía, etc.) exhiben intuitivamente la articulación de un estado despótico por medio de la equivalencia en la relación que encontramos en sus respectivos elementos (molino-aspas/déspota-súbditos) ${ }^{77}$;

d) en la exposición simbólica de un concepto de razón, la facultad de juzgar ejecuta pues una doble tarea: determina el objeto de acuerdo con el concepto que le corresponde (facultad de juzgar determinante), refiriendo a su vez ese objeto a una representación con la que no tiene una relación directa y cuyo objeto no es intuitivamente dable, pero al que expresa analógicamente. Es decir: en la exposición simbólica de una idea o concepto de razón, la referencia a la intuición se da por analogía con el procedimiento por el que la facultad de juzgar aprehende unitariamente una diversidad dada (la forma de la reflexión), i.e. la facultad de juzgar opera bajo la única restricción de hallar una representación intuitiva que exprese analógicamente aquello que escapa por definición a toda sensibilización cognoscitivamente válida ${ }^{78}$.

Ante la belleza de un objeto, i.e. la imposibilidad de hallar una representación bajo la que subsumir la forma de aquello que se nos presenta en la intuición, apercibimos la «conformidad a fin sin fin» (Zweckmäßigkeit ohne Zweck) de nuestras facultades representativas, esto es, el armónico funcionamiento de entendimiento e imaginación en cuanto facultades involucradas de manera necesaria en la producción de una representación unitaria de carácter general79.

El espontáneo juego entre el entendimiento y la imaginación, que en la experiencia de lo bello opera sin someterse a la regla de ningún concepto en particular, es análogo a la ausencia de determinación externa que es propia del concepto de libertad en sentido práctico, i.e. la capacidad para determinarse a obrar prescindiendo de estímulos externos. Ahora bien, de la misma manera que la libertad no implica carencia absoluta de regla sino la «conformidad a ley» de nuestro arbitrio en la toma de decisiones (en particular, el sometimiento a aquella ley que el sujeto racional se da a sí mismo), la imaginación se atiene al marco legal establecido por los principios trascendentales del entendimiento tanto en el enjuiciamiento como en la producción de la belleza ${ }^{80}$.

\footnotetext{
77 Cfr. KU, §59 (Ak, V, 352). Cabe encontrar multitud de tales exposiciones simbólicas en nuestro lenguaje ordinario y, en particular, en la mayor parte de términos metafísicos (v. gr. la definición lockeana del concepto de substancia como «portador» de los accidentes); íbid.

78 Íbid.

79 Cfr. I Einleitung KU (Ak, XX, 233).

80 Sobre este punto, cfr. Cohen, T., "Why Beauty is a Symbol of Morality?", en T. Cohen (ed.), Essays in Kant's Aesthetics, Chicago, The University of Chicago Press, 1982, pp. 232ss.
} 
De este modo, en la experiencia de la belleza se da una exposición simbólica de la idea de la libertad, esto es, se nos proporciona un referente intuitivo que patentiza analógicamente una representación que escapa por definición a toda sensibilización directa ${ }^{81}$.

\section{Conclusión}

Las escasas referencias al lenguaje en la obra de Kant son consistentes con la ubicación de las mismas allende el momento constituyente. El elemento lingüístico queda reducido a un mero instrumento con el que expresar nuestros pensamientos y referirse a los objetos «extralingüísticos». En este sentido afirmábamos que preguntarse por el significado de nuestros términos lingüísticos implica en Kant una reflexión sobre la naturaleza de nuestros conceptos y, en particular, acerca del fundamento de la relación particular-tipo (hipotiposis).

El parágrafo 59 de KU nos presenta los dos modos en que cabe entender toda posible relación de hipotiposis. En el dominio teórico, el fundamento de la relación entre el concepto (empírico o sensible puro) y un particular sensible determinado se halla en el esquema, y esto en un doble sentido:

a) el esquema de nuestros conceptos empíricos y sensibles puros, en tanto que representación de un procedimiento general por el que la imaginación puede proporcionar una imagen que corresponda a ese concepto, establece un conjunto suficiente de características que son parte de la representación global del objeto y cuyo cumplimiento por parte del particular sensible lo hace susceptible de ser subsumido bajo tal representación conceptual.

b) por otro lado, la posibilidad misma de toda relación particular-tipo reside en los esquemas trascendentales de la imaginación en cuanto determinaciones trascendentales del tiempo. Porque la posibilidad del conocimiento consiste en una determinación temporal del enlace de nuestras representaciones, los diversos modos en que es en general posible para nosotros determinar objetivamente la forma a priori originaria de la sensibilidad constituyen las características trascendentales de todo objeto posible y, en este sentido, aquellas condiciones involucradas en toda posible relación de hipotiposis en el dominio teórico.

En relación a aquellas representaciones -las ideas de la razón- que rehúyen toda sensibilización directa, su realidad objetiva depende en último término de la nocontradictoriedad del conjunto de notas o características que conforman su contenido. A pesar de su consistencia lógica, precisamente por no ajustarse a las condiciones a priori de la experiencia estas representaciones carecen de un significado cog-

81 Cfr. Guyer, P., Kant and the Experience of Freedom, New York, Cambridge University Press, 1993, p. 99 ss y p. 316. 
noscitivamente válido. El concepto de exposición simbólica responde a la pretensión de proporcionar cierto correlato sensible que haga aprehensible de un modo analógico aspectos de una representación no exponible directamente en la intuición.

\author{
Àlex Mumbrú Mora \\ Universidad de Barcelona \\ alex.mumbru@gmail.com
}

